\title{
Téoros
}

Revue de recherche en tourisme

\section{Femmes, pouvoir et tourisme}

Essai exploratoire

\section{Sylvie Gagnon}

Volume 16, numéro 3, automne 1997

Femmes et tourisme

URI : https://id.erudit.org/iderudit/1073320ar

DOI : https://doi.org/10.7202/1073320ar

Aller au sommaire du numéro

Éditeur(s)

Université du Québec à Montréal

ISSN

0712-8657 (imprimé)

1923-2705 (numérique)

Découvrir la revue

Citer cet article

Gagnon, S. (1997). Femmes, pouvoir et tourisme : essai exploratoire. Téoros, 16(3), 39-41. https://doi.org/10.7202/1073320ar

Ce document est protégé par la loi sur le droit d'auteur. L'utilisation des services d'Érudit (y compris la reproduction) est assujettie à sa politique d'utilisation que vous pouvez consulter en ligne.

https://apropos.erudit.org/fr/usagers/politique-dutilisation/
Cet article est diffusé et préservé par Érudit.

Érudit est un consortium interuniversitaire sans but lucratif composé de l'Université de Montréal, l'Université Laval et l'Université du Québec à Montréal. Il a pour mission la promotion et la valorisation de la recherche. https://www.erudit.org/fr/ 


\section{FEMMES, POUVOIR ET TOURISME}

\section{ESSAI EXPLORATOIRE}

\section{Sylvie Gagnon, consultante}

Les écrits féministes au Québec, qui traitent du pouvoir, s'intéressent surtout à la participation des femmes aux institutions politiques traditionnelles' (non sans remettre en question la définition même du pouvoir politique pour y inclure d'autres lieux informels de participation politique ${ }^{2}$ ) et, dans une moindre mesure, aux femmes cadres dans les organisations'. D'autres part, de façon plus ponctuelle et wofficielles, l'État, dans sa récente politique en matière de condition féminine, propose des mesures visant à accroûtre la participation des femmes dans les instances régionales (décentralisation oblige!). Ce sont ces constats qui ont orienté le contenu du présent article sur le pouvoir des femmes dans l'industrie du tourisme au Québec.

Ne pouvant prétendre à une réelle analyse féministe exhaustive (quand, comment et pourquoi?) de cette thematique, nous avons choisi de nous intéresser à la proportion de femmes (combien?) présentes actuellement dans les instances décisionnelles de l'industrie que sont Tourisme Québec, les associations touristiques régionales et les principales associations sectorielles. Nous jetterons également un bref coup d'oeil sur les femmes dans les entreprises touristiques.

\section{TOURISME QUÉBEC: LE PERDANT...}

Si nous pouvons nous targuer d'avoir eu deux femmes ministres pour diriger les destinés du tourisme au Québec (Claire Kirkland-Casgrain ${ }^{5}$ de 1970 à 1972 sous Bourassa et Rita Dionne-Marsolais de 1994 à 1997 dans le cabinet Parizeau/ Bouchard), un regard sur l'organigramme actuel de la nouvelle agence gouvernementale nous force à constater qu'une seule femme (Lucille Daoust, sous-ministre et directrice générale de l'agence) occupe un des quinze postes de la haute direction. Dans les differents ministères et organismes gouvernementaux la proportion de femmes à ces postes est de $28,1 \%^{6}$. A Tourisme Québec ce ratio est de $6,6 \% .$.

\section{LES ASSOCIATIONS TOURISTIQUES RÉGIONALES: GAGNANTES SAUF À MONTRÉAL...}

Les Associations touristiques régionales (ATR), ainterlocuteurs privilégies $\%$ de l'État en matière de tourisme, peuvent être comparées aux autres instances régionales (développement, santé, services sociaux, main-d'oeuvre) étudiées par le Secrétariat à la condition féminine dans le cadre de son analyse sur la place des femmes dans le développement des régions.

Sur l'ensemble des 18 ATR du Québec, deux femmes seulement occupent des postes de direction $(11,1 \%)$. Par contre une association sur trois est présidée par une femme. Elles constituent (incluant les présidentes) $28,6 \%$ des comités exécutifs et, au total, $32 \%$ de l'ensemble des 18 conseils d'administration.
Les associations touristiques régionales de Manicouagan, des Laurentides et de la Mauricie-Bois-Francs sont les seules ATR qui comptent une proportion ếgale ou superieure de femmes dans leur conseil d'administration. Au niveau de l'exécutif, trois ATR seulement présentent un conseil exécutif composế d'autant de femmes que d'hommes. Il s'agit des associations touristiques de Duplessis, de la MauricieBois-Francs et de la Gaspésie.

L'association touristique régionale de la Mauricie-Bois-Francs est de loin l'association où les femmes sont les plus nombreuses (même la direction est occupée par une femme) avec $64 \%$ de représentativité à son conseil d'administration. À l'opposé, la région de Montréal est la moins performante avec $12,5 \%$ de femmes seulement. Cette région, tout comme celle de Charlevoix, ne compte aucune femme au sein de son comité exécutif.

\section{DES LEÇONS À DONNER AUX CONSEILS RÉGIONAUX DE DEVELOPPEMENT}

Comparativement aux CDR, les ATR font bonne figure au chapitre de la représentation des femmes. Les comités exécutifs et les conseils d'administration des CRD comptent seulement une femme sur cinq en moyenne comparativement à près d une sur trois pour les ATR. Dix des seize CDR ont des conseils d'administration où siègent moins de $20 \%$ de femmes comparativement à six ATR sur dix-huit.

Tout comme pour les ATR, la région où la représentation des femmes est la plus élevée est la région Mauricie-Bois-Francs. A l'instar de Montréal, la région Gaspésie- 
Iles-de-1a-Madeleine ne compte aucune femme sur son conseil exécutif. La CôteNord est la région qui compte le moins de femmes à son conseil d'administration avec $12,5 \%$ seulement.

La présence des femmes au sein des centres régionaux de la Société québécoise de développement de la main-d'oeuvre (SQDM) ainsi que des Régie régionale de la santé et des services sociaux (RRSSS) est comparable à celle des femmes au niveau des ATR. La SQDM compte en moyenne $37 \%$ de femmes parmi ses membres et les RRSSS $34 \%$. Les commissions scolaires par contre ont une représentation féminine supérieure aux autres instances régionales: $39 \%$ de femmes les président et plus de $45 \%$ oceupent des postes à titre de commissaires. L'éducation étant un des métiers traditionnels des femmes, une représentation plus forte dans ce domaine n'est pas surprenante.

\section{LES ASSOCIATIONS SECTORIELLES: UNE PRÉSENCE MOINS MARQUÉE}

Il existe au Québec plus d'une cinquantaine d'associations sectorielles dans l'in-

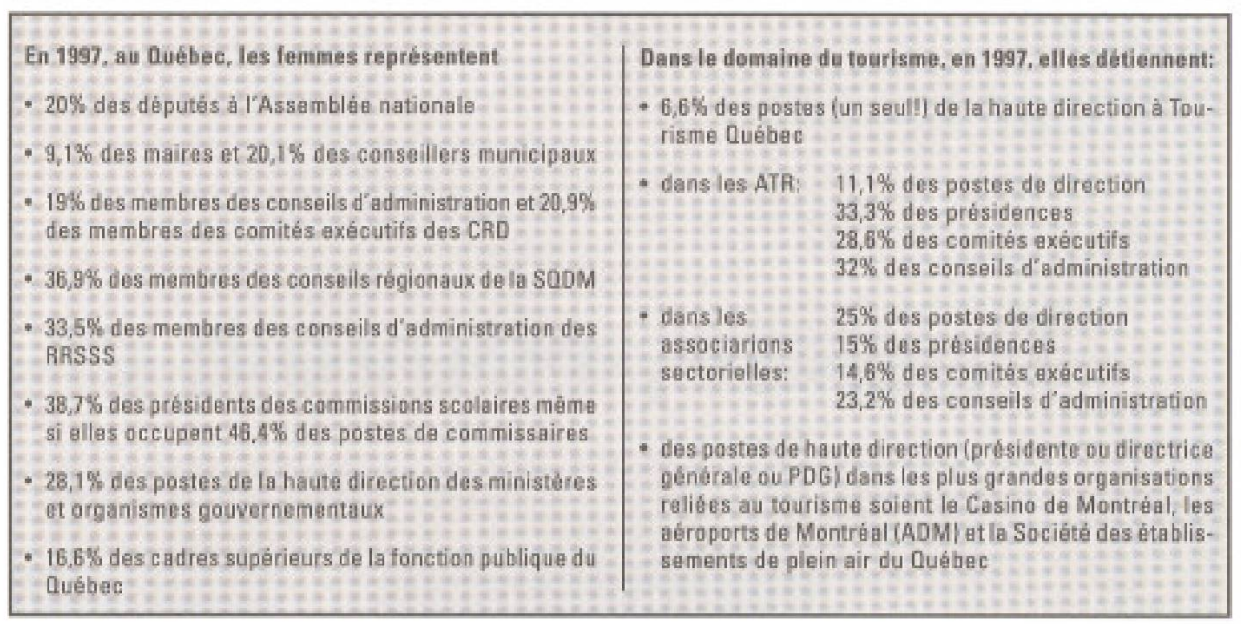

dustrie du tourisme selon le répertoire de Tourisme Québec. Nous avons retenus uniquement celles qui regroupent des entreprises touristiques ou représentent des produits touristiques majeurs.

A l'exception des postes de direction, les femmes se retrouvent moins bien représentées dans les associations sectorielles que dans les associations touristiques régionales. Au niveau des comités exécutifs par exemple, on retrouve presque deux fois plus de femmes dans les ATR que dans les associations sectorielles. Au niveau des conseils d'administration les associade femmes par rapport au $32 \%$ qui siègent sur ceux des ATR.

Les associations sectorielles comptent $25 \%$ de directrices et $15 \%$ de présidentes. Par rapport aux ATR, ces proportions sont inversées; les associations touristiques régionales comptent moins de femmes directrices que de femmes présidentes. Les conseils d'administration sont composés de $23 \%$ de femmes et les comités exécutifs de $14,6 \%$. tions sectorielles ne comptent que $23,2 \%$

\section{TABLEAU 1}

Les femmes à la direction et aux conseils d'administration des associations touristiques régionales en 1997

\begin{tabular}{|c|c|c|c|c|c|c|c|c|}
\hline \multirow{2}{*}{$\begin{array}{l}\text { Direction } \\
\text { H:homme } \\
\text { F: femme }\end{array}$} & \multirow[t]{2}{*}{ ATR } & \multicolumn{4}{|c|}{ Comité exécutif } & \multicolumn{3}{|c|}{ Conseil d'administration } \\
\hline & & $\begin{array}{l}\text { Présidence } \\
\text { H:homme } \\
\text { F: femme }\end{array}$ & $\begin{array}{l}\text { Nbre de } \\
\text { membres }\end{array}$ & $\begin{array}{l}\text { Nbre de } \\
\text { femmes }\end{array}$ & $\%$ & $\begin{array}{l}\text { Nbre de } \\
\text { membres }\end{array}$ & $\begin{array}{l}\text { Nbre de } \\
\text { femmes }\end{array}$ & $\%$ \\
\hline $\mathrm{H}$ & Iles-de-la-Madeleine & $\mathrm{F}$ & 4 & 1 & $25,0 \%$ & 7 & 2 & $28,6 \%$ \\
\hline $\mathrm{H}$ & Gaspésie & $\mathrm{H}$ & 3 & 2 & $66,7 \%$ & 8 & 3 & $37,5 \%$ \\
\hline $\mathrm{H}$ & Bas-St-Laurent & $\mathrm{F}$ & 5 & 1 & $20,0 \%$ & 14 & 6 & $42,9 \%$ \\
\hline $\mathrm{H}$ & Québec & $\mathrm{F}$ & 6 & 1 & $16,7 \%$ & 23 & 6 & $26,1 \%$ \\
\hline $\mathrm{H}$ & Charlevoix & H & 5 & 0 & $0,0 \%$ & 13 & 5 & $38,5 \%$ \\
\hline $\mathrm{H}$ & Chaudiere-Appalaches & H & 7 & 3 & $42,9 \%$ & 22 & 7 & $31,8 \%$ \\
\hline $\mathrm{F}$ & Mauricie-Bois-Francs & $\mathrm{F}$ & 6 & 3 & $50,0 \%$ & 14 & 9 & $64,3 \%$ \\
\hline $\mathrm{H}$ & Cantons de l'Est & $\mathrm{H}$ & 5 & 2 & $40,0 \%$ & 6 & 2 & $33,3 \%$ \\
\hline $\mathrm{H}$ & Montérégie & H & 6 & 1 & $16,7 \%$ & 19 & 7 & $36,8 \%$ \\
\hline $\mathrm{H}$ & Lanaudière & H & 6 & 1 & $16,7 \%$ & 24 & 4 & $16,7 \%$ \\
\hline $\mathrm{H}$ & Laurentides & $H$ & 6 & 2 & $33,3 \%$ & 18 & 9 & $50,0 \%$ \\
\hline $\mathrm{H}$ & Montréal & H & 6 & 0 & $0,0 \%$ & 16 & 2 & $12.5 \%$ \\
\hline H & Outaouais & $\mathrm{H}$ & 5 & 1 & $20,0 \%$ & 16 & 3 & $18,8 \%$ \\
\hline $\mathrm{H}$ & Abitibi-Témiscamingur & $\mathrm{F}$ & 6 & 2 & $33,3 \%$ & 14 & 4 & $28,6 \%$ \\
\hline $\mathrm{H}$ & Saguenay-Lac-St-Jean & $H$ & 6 & 1 & $16,7 \%$ & 27 & 5 & $18,5 \%$ \\
\hline $\mathrm{H}$ & Manicouagan & $\mathrm{F}$ & 6 & 2 & $33,3 \%$ & 18 & 9 & $50,0 \%$ \\
\hline$H$ & Duplessis & H & 6 & 3 & $50,0 \%$ & 11 & 3 & $27,3 \%$ \\
\hline$F$ & Laval & $H$ & 6 & 2 & $33,3 \%$ & 15 & 2 & $13,3 \%$ \\
\hline $\begin{array}{ll}2 \mathrm{~F} & 11,1 \% \\
16 \mathrm{H} & 88,8 \%\end{array}$ & Total & $\begin{array}{l}6 \mathrm{~F} \\
(33,3 \% \%)\end{array}$ & 100 & 28 & $28, \overline{6} \%$ & 285 & 88 & $32,0 \%$ \\
\hline
\end{tabular}


Au niveau du comité exécutif, c'est à l'Association hôtelière de la région de Québec et chez Tourisme Jeunesse que les femmes sont les mieux représentées $(50 \%)$. Par contre, les femmes sont totalement absentes des comités exécutifs d'une dizaine de secteurs. En contrepartie deux exécutifs comptent $50 \%$ de femmes.

Au niveau des conseils d'administration ce sont ceux de Tourisme-Jeunesse et d'ACTA-Québec qui comptent les plus fortes proportion de femmes tandis que trois associations ne comptent aucune femme ni à la direction, ni à leur conseil d'administration: 1'Association des stations de ski, la Fédération des pourvoyeurs du Québec et la Fédération des clubs de motoneigistes du Québec.

\section{CONCLUSION: QUAND ON SE COMPARE, ON SE CONSOLE...}

Si on peut s'étonner du peu de femmes à la haute de direction de Tourisme-Québec, on ne peut passer sous silence la place relativement considérable, par rapport aux autres organismes, qu'occupent les femmes dans les instances rếgionales touristiques. Les ATR regroupant elles aussi principalement, tout comme les associations sectorielles, des entreprises privées, on peut conclure que les femmes entrepreneurs et les femmes directrices d'entreprises ont davantage droit de cités dans les régions que dans les grands centres (comme Montréal...) et dans les associations représentant l'ensemble du territoire québécois.

Quand on observe que dans les programmes de formation en tourisme et en hôtellerie au Québec de tous les niveaux d'enseignement, les femmes sont largement majoritaires on ne peut qu'être convaincu que, demain, elles prendront encore plus de place dans les différentes sphères politiques et économiques du domaine touristique. Nous le souhaitons!

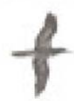

\section{NOTES}

1 Voir Chantal Maille (1990), Evelyne Tardy (1982) et plusieurs écrits de Manon Tremblay (de 1989 à 1996), toutes trois spécialistes de la question.

2 Chantal Maille (1990), Les québecoises et la conquête du pouvoir politique, pp 8-9

3 Voir entres autres Claudine Baudoux, Claire V. de la Durantaye (1988) et Irène Lépine, Carole Simard (1991)

4 Gouvernement du Québec (1997), Programne d'action 1997-2000 pour toutes les Quebécoises, Secrétariat à la condition féminine, $138 \mathrm{p}$.

5 Claire Kirkland-Casgrain, en 1961, fut la première femme élue députée à l'Assemblée nationale

6 Toutes les données présentées dans ce texte (a l'exception de celles sur les ATR et les associations sectorielles compilées par nous) sont tirées du document La place des femmes dans le développement des régions (1997) produit par le gouvernement du Québec dans le cadre de la politique en matière de condition féminine.

TABLEAU 2

Les femmes à la direction et aux conseils d'administration des associations sectorielles en 1997

\begin{tabular}{|c|c|c|c|c|c|c|c|c|}
\hline \multirow{2}{*}{$\begin{array}{l}\text { Direction } \\
\text { H:homme } \\
\text { F: femme }\end{array}$} & \multirow{2}{*}{$\begin{array}{l}\text { Associations } \\
\text { sectorielles }\end{array}$} & \multicolumn{4}{|c|}{ Comité exécutif } & \multicolumn{3}{|c|}{ Conseil d'administration } \\
\hline & & $\begin{array}{l}\text { Présidence } \\
\mathrm{H} \text { :homme } \\
\mathrm{F} \text { : femme }\end{array}$ & $\begin{array}{l}\text { Nbre de } \\
\text { membres }\end{array}$ & $\begin{array}{l}\text { Nbre de } \\
\text { femmes }\end{array}$ & $\%$ & $\begin{array}{l}\text { Nbre de } \\
\text { membres }\end{array}$ & $\begin{array}{l}\text { Nbre de } \\
\text { femmes }\end{array}$ & $\%$ \\
\hline $\mathrm{F}$ & ACTA-QUÉ⿴囗十 & $\mathrm{H}-\mathrm{F}$ & 5 & 2 & $40,0 \%$ & 15 & 7 & $46,7 \%$ \\
\hline H & Ass. des hốtaliers de la province dé Québect & $\mathrm{H}$ & 6 & 0 & $0,0 \%$ & 18 & 1 & $5,6 \%$ \\
\hline H & ÁEs. đQs hớtels đu Grand Mantréal & $\mathrm{H}$ & 3 & 0 & $0,0 \%$ & 11 & 1 & $9,1 \%$ \\
\hline H & Ass. hâtelière de la rógian de Quèber & $\mathrm{H}$ & 4 & 2 & $50,0 \%$ & 12 & 3 & $25,0 \%$ \\
\hline H & Ass, des prod. en tourisme d'avanture & $\mathrm{H}$ & 3 & 0 & $0,0 \%$ & 10 & 1 & $10,0 \%$ \\
\hline H & Ass. des proprietaires de golf du Quéhec & $\mathrm{H}$ & 5 & 0 & $0,0 \%$ & 12 & 3 & $25,0 \%$ \\
\hline H & Asss. des nestauratours du Quábec & $\mathrm{F}$ & 3 & 0 & $0,0 \%$ & 11 & 3 & $27,3 \%$ \\
\hline H & Ass, des stations de ski du Quethèc & $\mathrm{H}$ & 4 & 0 & $0,0 \%$ & 7 & 0 & $0,0 \%$ \\
\hline$F$ & Ass. des terrains de camping du Queberc & $\mathrm{H}$ & 5 & 1 & $20,0 \%$ & 15 & 3 & $20,0 \%$ \\
\hline H & Ass. québécaise dies transporteurs aéniens & $\mathrm{H}$ & - & $=$ & - & 8 & 2 & $25,0 \%$ \\
\hline H & Chaire de tourisme & - & $=$ & - & - & 9 & 3 & $33,3 \%$ \\
\hline $\mathrm{F}$ & CORHT & $H$ & 4 & 1 & $25,0 \%$ & 19 & 6 & $31,6 \%$ \\
\hline $\mathrm{F}$ & Féd des agricotours du duábec & $\mathrm{H}$ & 4 & 0 & $0,0 \%$ & 19 & 5 & $26,3 \%$ \\
\hline H & Fád. des paurvopeurs du Québec inc. & $H$ & 5 & 0 & $0,0 \%$ & 18 & 0 & $0,0 \%$ \\
\hline$F$ & Féd. quéthécnise des uffices de touriame & $\mathrm{H}$ & 5 & 2 & $40,0 \%$ & 13 & 4 & $30,8 \%$ \\
\hline H & Tourisme Jaunถรse & $\mathrm{F}$ & 4 & 2 & $50,0 \%$ & 8 & 5 & $62,5 \%$ \\
\hline H & Sec. des attractions touristiques du De & $\mathrm{H}$ & 3 & 0 & $0,0 \%$ & 13 & 4 & $30,8 \%$ \\
\hline H & Soc. des fites et fustivals du Québer: & $\mathrm{H}$ & 7 & 1 & $14,3 \%$ & 14 & 5 & $35,7 \%$ \\
\hline H & ATRAO & $\mathrm{H}$ & 6 & 1 & $16,7 \%$ & 19 & 6 & $31,6 \%$ \\
\hline $\mathrm{H}$ & Fád. des cluts de mâtonteigistes du Dc & $\mathrm{H}$ & 6 & 0 & $0,0 \%$ & 16 & 0 & $0,0 \%$ \\
\hline $\begin{array}{l}5 F \\
(25 \%) \\
15 \mathrm{H} \\
(75 \%)\end{array}$ & Total & $\begin{array}{l}3 F \\
(15 \%)\end{array}$ & 82 & 12 & $14,6 \%$ & 267 & 62 & $23,2 \%$ \\
\hline
\end{tabular}

Source: Donneg obtenues par interviews télephoniques auprès des associations (eutomne 1997) 